\title{
Modèle gravimétrique régional de l’intrusif du Mont Mégantic
}

\author{
Maurice K. Seguin*, Janusz Frydecki* et Sylvie Roy \\ Laboratoire de géophysique, Département de géologie, Université Laval, Québec G1K 7P4, Canada
}

\author{
Date Received March 15, 1988 \\ Date Accepted October 5, 1988
}

\begin{abstract}
Une approche non classique de la modélisation du champ gravimétrique régional est présentée. Le concept mis de l'avant dans la méthode de Griffin permettant d'extraire l'anomalie résiduelle est celui qui s' apparente le plus à l'approche proposée dans cet article. La tendance actuelle fait surtout appel à des fonctions mathématiques et à l'analyse numérique. La méthode proposée est comparée avec d'autres techniques de représentation de la régionale en prenant comme exemple la région du Mont Mégantic. Les résultats suivants sont présentés: (1) approximation du champ régional par les polynômes du ler, 2ième et 3ième degré, (2) approximation du champ régional par la méthode de la moyenne mobile suivant quelques versions de fenêtre différentes, (3) corrélations des paramètres gravimétriques et topographiques, (4) approximation des cartes de contour et des modèles tridimensionnels pour chaque version calculée, (5) comparaison des résultats obtenus et choix méthodologiques.
\end{abstract}

A non-classical approach towards regional, gravity-field modelling is presented, which is closest to the concept put forward within Griffin's method of isolating the residual anomaly. Current trends mainly call upon mathematical functions and numerical analysis. The Mount Megantic area is used as an example to compare the proposed method with other techniques of representing the regional trend. Results are (1) approximation of the regional field with the help of linear, quadratic and cubic polynomials; (2) approximation of the regional field through the moving-average method using a few different windows; (3) correlation of gravity and terrain parameters; (4) approximation of contour maps and three-dimensional models for each calculated version; and (5) comparison of the results and methodological choices

\section{INTRODUCTION}

Un des problèmes majeurs rencontrés dans l'interprétation des données gravimétriques est relié à la définition du champ régional (anomalie gravimétrique régionale). La résolution et la qualité de l'anomalie régionale constituent une condition primordiale afin de bien distinguer l'anomalie résiduelle utilisée pour obtenir une image bi- oú tridimensionnelle descorps géologiques camouflés dans la croûte terrestre. Un exemple de cas particulièrement compliqué de définition de la régionale a été rencontré au Mont Mégantic. Afin d'obtenir une régionale, il s'est avéré nécessaire de comparer diverses techniques de modélisation et d'en concevoir une, suffisamment versatile pour arriver à une solution optimale.

\section{CADRE GEOLOGIQUE}

Dans la région de Mont Mégantic située environ $150 \mathrm{~km}$ à l'est de Montréal, l'intrusif du Mont Mégantic constitue une aire à relief dominant et bien visible, dont l'altitude atteint $1120 \mathrm{~m}$ audessus du niveau de la mer. La géologie du Mont Mégantic (Fig. 1a) a déjà été décrite par plusieurs auteurs (Burton, 1931; McGerrigle, 1934; Reid, 1960, 1976; Chevé, 1975, 1977, 1978; Clément et De Kimpe, 1977; Danis, 1984; Seguin et St-Hilaire,
1985). L'intrusif du Mont Mégantic qui recoupe la formation de Compton recouvrant presque tout le territoire de cette région est composé de granites affleurant au centre sud du mont, de gabbro et de syénite qui entourent partiellement le centre granitique. Le problème géologique posé a trait à l'hypothèse des intrusions multiplesqui a déjà été confirmée par des études paléomagnétiques (Seguin et St-Hilaire, 1985). Il manque toutefois un modèle géométrique de l'intrusif (ou bien des intrusifs) et de la rochehôte à proximité du mont. A cette fin, on a effectué en 1985 et 1987 deux campagnes gravimétriques dont les premiers résultats furent présentés (Roy et Seguin, 1986; Frydecki, Seguin et Roy, 1987). La Figure $1 \mathrm{~b}$ montre la distribution des points de mesures gravimétriques sur et au pourtour du Mont Mégantic.

\section{ANALYSE DE BOUGUER}

L'image schématisée, simplifiée et généralisée en deux et trois dimensions de l'anomalie de Bouguer sur et au pourtour du Mont Mégantic après corrections topographiques apparaît à la Figure 2. L'analyse détaillée des données de terrain, de leur qualité, distribution et diversité a permis d'utiliser la plupart des observations pour arriver à la préparation des cartes du champ régional.

*Groupe de Recherche en Géochimie et Géophysique Appliquées 


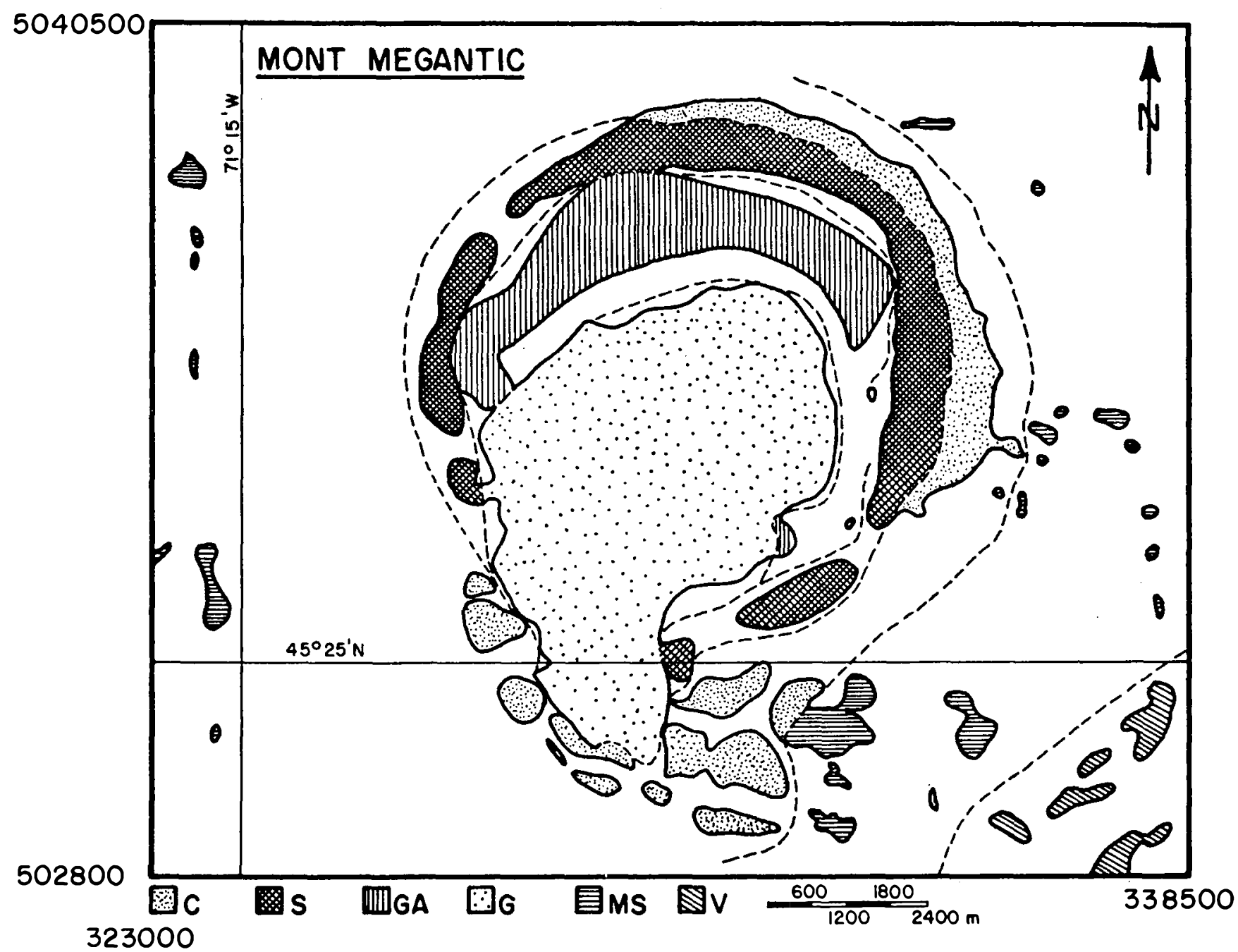

Fig. 1a. Cadre géologique du Mont Mégantic (modifié d'après Reid, 1976). S - syénite, Ga - gabbro, G - granite, Ms - roches métasédimentaires, $\mathrm{V}$ - roches volcaniques.

La Figure 3 illustre la carte de contour détaillée des anomalies de Bouguer sur le Mont Mégantic.

Après cette étape, les anomalies locales doivent être isolées. Celles-ci pourront servir à l'obtention des modèles tridimensionnels du Mont Mégantic. Pour atteindre ce but, il faut que les données soient uniformément représentées sur chacun des points saillants des objets modélisés et, pour des raisons statistiques, la quantité des données couvrant chaque objet doit être suffisamment représentative. La distribution d'information doit être cohérente, homogène et conforme à la loi de Gauss; la gamme de valeurs de données doit être bien étendue dans l'aire de chaque objet modélisé.

On a constaté que ces exigences ont été suffisamment remplies grâce à une deuxième campagne de mesure (en 1987) pour effectuer des travaux d'interprétation de détail.

\section{CORRELATION DES PARAMETRES}

Les corrélations des paramètres gravimétriques vs autres paramètres observés constituent une partie importante de nos travaux de recherche. On a déjà présenté (Roy et Seguin, 1986) les corrélations des susceptibilités vs densités, et (Frydecki et al., 1987) une corrélation des valeurs de Bouguer (A.B) vs d'altitude
(H) de la station de mesure. Si on tient compte des limites du nuage de points dans cette analyse (Fig. 4), on note deux tendances directionnelles principales qui sont significativement différentes. On observe deux tendances directionnelles principales (Fig. 4) qui constituent les limites du nuage de points dans cette analyse. Les résultats numériques obtenus par approximation du 2ème degré et en 3 dimensions sont les suivants:

Pour l'extrême le plus élevé,

A.B. $=-48.87+4.19199 x-2.39791 \mathrm{y}+0.0402576 \mathrm{x}^{2}+$
$0.0334497 \mathrm{y}^{2}+0.01431012 \mathrm{H}(\mathrm{mGal})$ et pour l'extrême le plus bas:

A.B.
$0.0156506 \mathrm{y}^{2}-0.01438978 \mathrm{H}(\mathrm{mGal})$

Les unités des coordonnées $x$ et $y$ utilisées pour ces calculs ont été définies d'après le système UTM de la manière suivante:

$$
\begin{aligned}
& {[x]=0.002 *(x U T M-306000 \mathrm{~m} \mathrm{E})} \\
& {[y]=0.002 *(y U T M-501200 \mathrm{~m} \mathrm{~N})}
\end{aligned}
$$




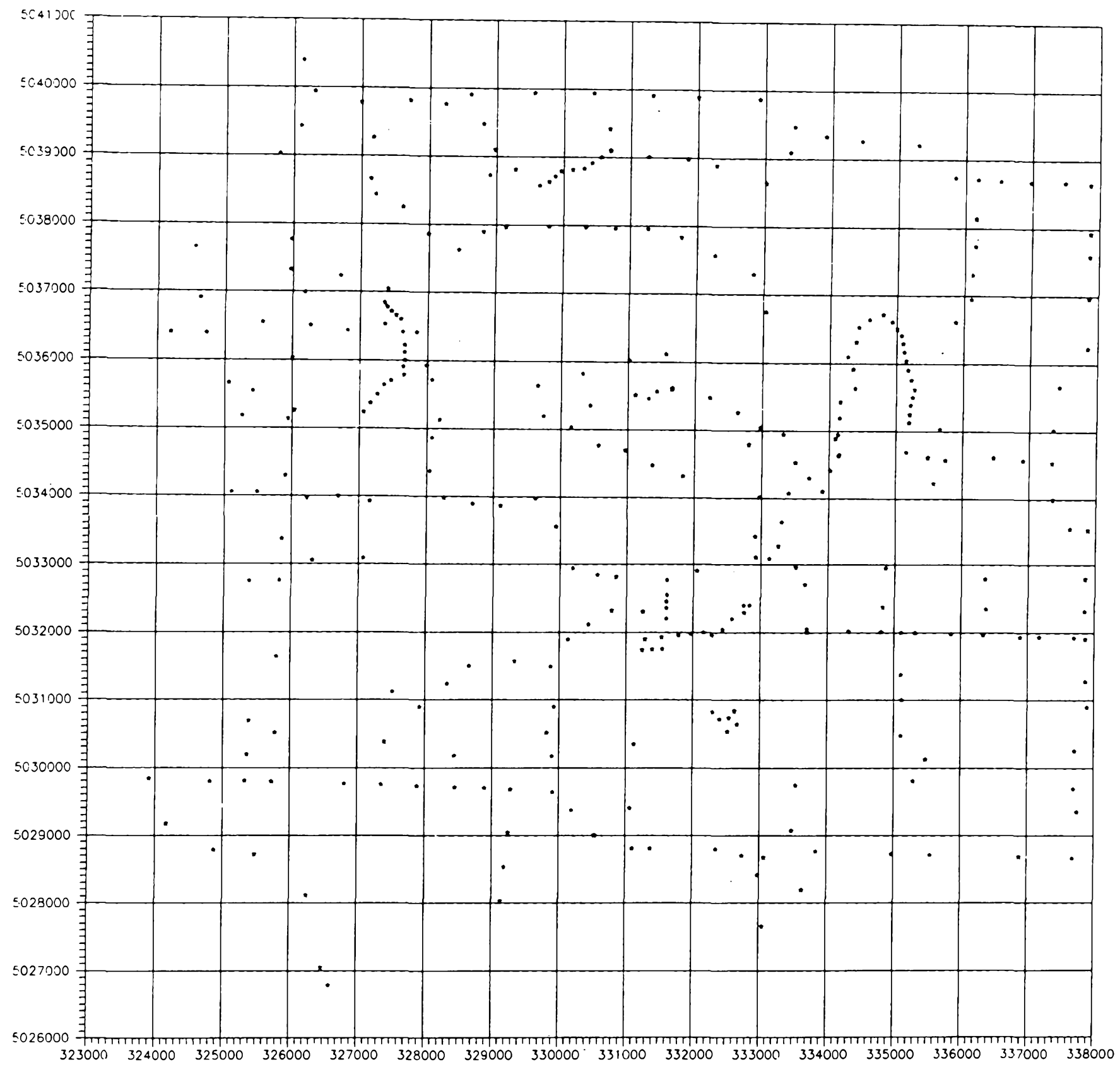

Fig. 1b. Répartition des stations de mesure gravimétrique sur et au pourtour du Mont Mégantic.

Comme on estime des tendances directionnelles des valeurs limites, une évaluation des erreurs quadratiques moyennes n'a aucune signification puisque la notion de limite (extrême) va à l'encontre de celle de la moyenne. Par contre, une erreur quadratique moyenne peut être obtenue pour la tendance directionnelle caractéristique d'une formation géologique spécifique. Les densités des formations (selon les méthodes de Nettleton, par exemple) sont-elles identifiables avec les densités extrêmes? La plus élevée est de l'ordre de $3.01 \mathrm{~g} \mathrm{~cm}^{-3} \mathrm{et}$ la plus basse de l'ordre de $2.33 \mathrm{~g} \mathrm{~cm}^{-3}$. Les relations sont visibles mais il faut distinguer chaque formation séparément, (Seguin et Frydecki, 1989).

Le nuage de points entre les deux limites réflète la complexité de la géologie ce qui suggère la présence de plusieurs (+ que 2) formations géologiques caractérisées par des densités distinctives et en conséquence des tendances directionnelles différentes.

\section{CHAMP REGIONAL}

\section{(1) Idée maîtresse}

Le taux de succès dans le domaine de l'interprétation des données gravimétriques dépend du modèle régional choisi et appliqué. Il existe plusieurs approches et chaque fois le choix doit être conforme aux contexte et style géologiques, à la tectonique et surtout à l'échelle du problème envisagé et aux dimensions de l'objet modélisé. Pour les travaux à l'échelle globale, les évènements et cibles géologiques de la dimension d'un canton par exemple ont un caractère local et doivent être éliminés. Au contraire, pour un objet comme le Mont Mégantic, cette dimension est considérée comme vaste et vraiment régionale. 

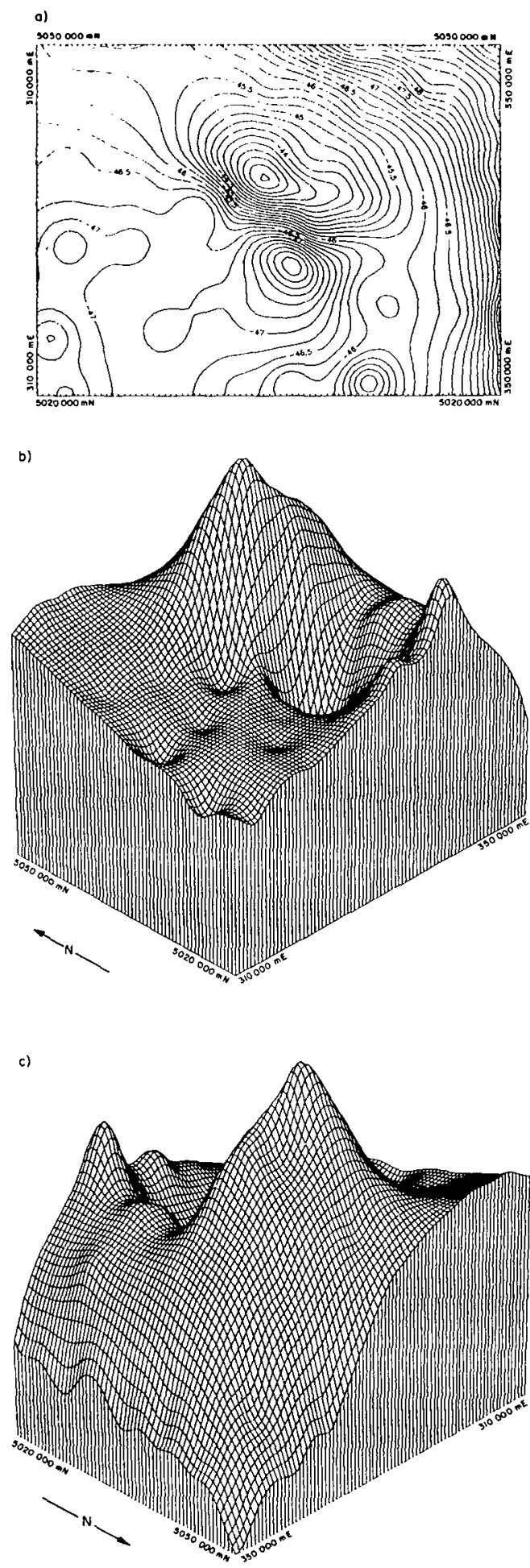

Fig. 2. (a) Représentation bidimensionelle des anomalies (+ et -) de Bouguer (A.B.) du Mont Mégantic (au centre) et des unités géologiques encaissantes sur le pourtour. (b) Représentation tridimensionnelle des (A.B.) de la même région (après rotation de $315^{\circ}$ en sens horaire suivant la verticale et rotation de $15^{\circ}$ suivant l'horizontale). L'anomalie positive à l'arrière plan correspond à la bande de gabbro au nord et l'anomalie négative à l'avant plan au granite plus au sud. (c) Même description qu'en (b) excepté que l'angle de rotation verticale est de $135^{\circ}$ en sens horaire. L'anomalie positive à l'avant plan représente le gabbro et un fragment de la syénite.
Ainsi donc, le choix de la méthode employée découle de l'analyse d'échelle du problème, soit de l'échelle de l'objet à modéliser et, ce qui est tout aussi important, de l'échelle des travaux (mesures) de terrain ainsi que de la procédure analytique. L'implantation des techniques d'ordinateur dans l'interprétation géophysique et pour l'interprétation gravimétrique en particulier a conduit au développement des méthodes hautement mathématisées telles les polynômes de différents degrés, les séries de Fourier, les filtres de fréquence très en vogue actuellement, etc. Ces approches sont excellentes mais elles impliquent toujours une interrogation; par exemple, quel degré faut-il choisir pour la méthode polynomiale? $1^{\text {er }}, 2^{\text {ème }}$ ou $3^{\text {emec }}$ Quel critère vaut-il mieux utiliser pour faire ce choix? Pour de simples cartes de contour, il est évident qu'il faut itérer jusqu'à l'obtention d'un minimum d'écart si l'on fait appel à la méthode des moindres carrés ou à une autre méthode similaire. Mais pour la régionale, il ne s'agit pas d'une interpolation mais plutôt de l'approximation d'un modèle conceptualisé et avec une échelle présélectionnée. C'est pourquoi les anciennes méthodes telles celles de Griffin (1949), Saxov et Nygaard (1953), Nettleton (1954) et autres, étaient vraiment justifiées de par les dimensions et échelles exigées et appliquées. Le choix d'une méthode optimale dépend aussi de la méthode choisie pour l'analyse des données. En utilisant un polynôme ou une moyenne mobile, on n'obtient pas de renseignements relatifs au contenu spectral mais par contre l'analyse spectrale ne donne pas d'information concernant la dimension des objets étudiés. Donc, si on utilise une méthode, on n'obtient pas l'information fournie par une autre méthode.

\section{(2) Polynômes}

Très souvent, on utilise les polynômes pour effectuer les approximations des observations provenant de levés géophysiques. C'est un outil efficace et, en utilisant l'ordinateur, très facile à appliquer. L'application des polynômes orthogonaux de Tchebyschev donne une occasion unique d'harmoniser l'image obtenue à la réalité. Mais le but de la régionale n'est justement pas la représentation exacte d'un objet géologique spécifique de sorte que le choix du degré d'un polynône joue un rôle prédominant.

Dans la région étudiée, on a essayé trois possibilités: $1^{\text {er }}, 2^{\text {żme }}$ et $3^{\text {ème }}$ degré. Chacun de ces cas est probablement suffisamment général pour pouvoir imaginer que les détails (anomalies locales, bruits, etc.) seront éliminés et que tout ce qui reste correspond aux images d'évènements et cibles géologiques à grande échelle. Pour ce faire, on a utilisé quelque 111 valeurs additionnelles de A.B. représentant l'anomalie de Bouguer en dehors de la région considérée, données obtenues de la Commission géologique du Canada. Ces valeurs permettent de calculer le champ régional généralisé autour du Mont Mégantic.

Les résultats d'approximation par le polynôme du $2^{\text {eme }}$ degré de type $z=a_{0}+a_{1} x+a_{2} y+a_{3} x^{2}+a_{4} y^{2}+a_{5} x y$ sont présentés à la Figure 5 et ceux d'approximation par le polynôme du $3^{\text {eme }}$ degré du type $=a_{0}+a_{1} x+a_{2} y+a_{3} x^{2}+a_{4} y^{2}+a_{5} x y+a_{6} x^{3}+a_{1}$ $y^{3}$ à la Figure 6. On a considéré le polynôme de $1^{\text {er }}$ degré trop général pour être directement applicable à notre échelle mais, de toute évidence, cette approximation représente aussi très bien les 


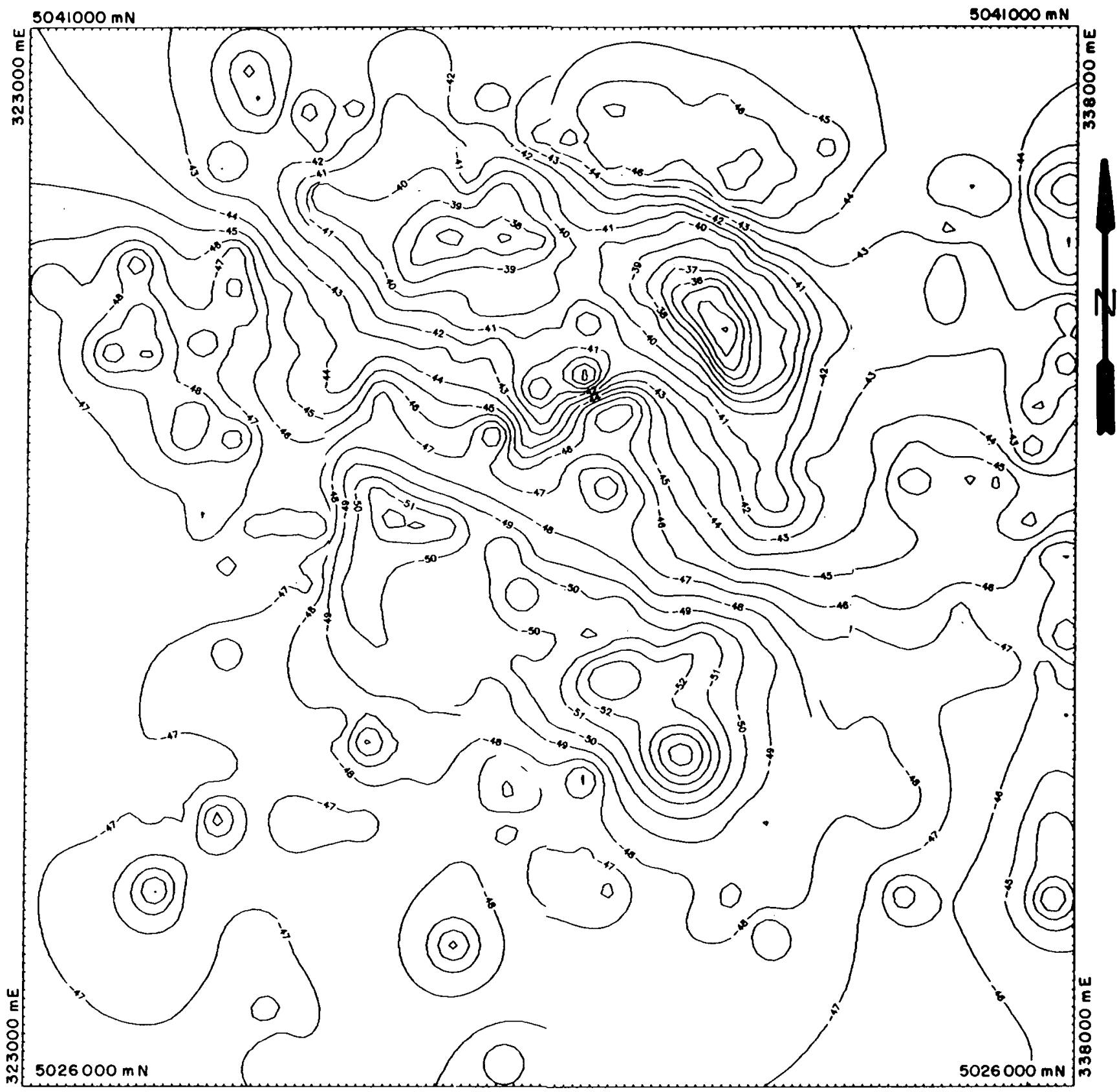

Fig. 3. Carte détaillée des isocontours des anomalies de Bouguer sur et au pourtour du Mont Mégantic. Intervalles de contour: $1 \mathrm{mGal}$. Les limites de la carte sont les mêmes que sur la Figure 1.

tendances directionnelles à une échelle globale. C'est pourquoi, chacune des approximations présentées est superposée à l'approximation du $1^{\text {er }}$ degré. On observe des différences entre ces deux (ou bien trois) images surtout à proximité des bordures de la superficie analysée. Les valeurs de la régionale sont plus ou moins les mêmes à l'intérieur du territoire du Mont Mégantic. Il existe une seule grande différence dans les directions de variations du champ; cette différence est une fonction de la méthode d'approximation; plan incliné (1 $1^{\text {er }}$ degré), parabolique ( $2^{\text {ème }}$ degré) et enfin en forme de selle typique pour le $3^{\text {ème }}$ degré (Fig. 7). Le choix entre le $2^{\text {ème }}$ et le $3^{\text {ème }}$ degré est difficile. Selon la tradition, il faudrait probablement choisir l'approximation du $3^{\text {ème }}$ degré, mais ce choix n'est pas justifié par l'objet géologique, l'échelle de travail ou tout autre critère objectif. Excellente du point de vue technique, la méthode des polynômes, comme les autres méthodes artificielles, n'a pratiquement aucune explication soit physique soit géologique.

\section{(3) Résultats numériques}

Les approximations de la régionale gravimétrique sont présentées pour les polynômes de degré 1,2 et 3 . Approximation du $1^{\text {er }}$ degré:

$$
\text { A.B. }{ }_{\text {reg }}=-43.12-0.09099 x-0.020407 y(m G a l)
$$

Approximation de $2^{\text {ème }}$ degré: 


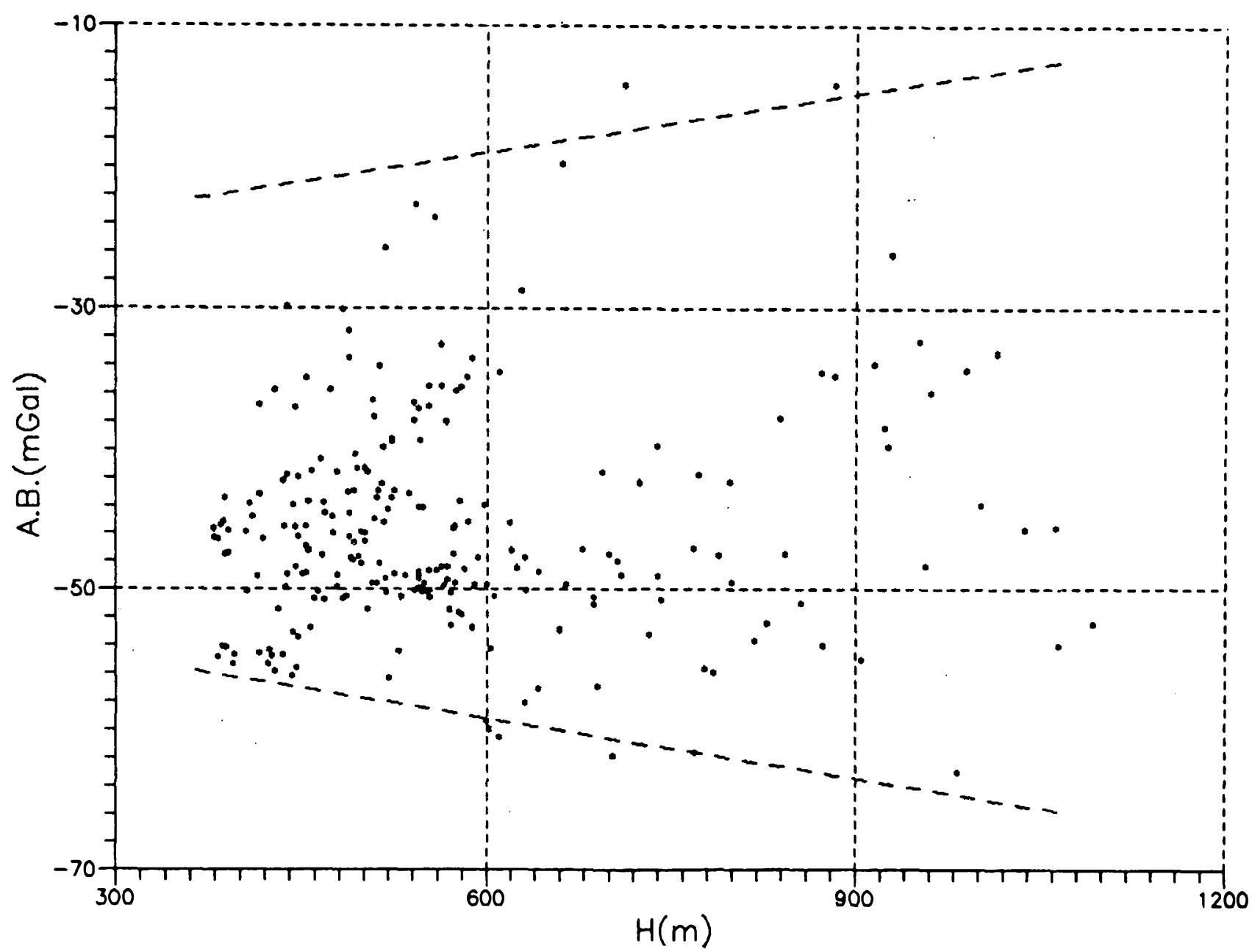

Fig. 4. Comparaison des anomalies de Bouguer vs altitude. Les tendances directionnelles représentant les valeurs extrêmes (limites) sont indiquées.

A.B. ${ }_{{ }_{\text {rg }}}=-42.34-0.079857 \mathrm{x}-0.082695 \mathrm{y}+0.00049931 \mathrm{x}^{2}+$ $0.00079178 \mathrm{y}^{2}-0.00092456 \mathrm{xy}(\mathrm{mGal})$

Approximation du $3^{\text {ème }}$ degré:

A.B. $=-47.55+0.51197 x-0.33476 y-0.0096933 x^{2}+$ $0.007392 y^{2}-0.0022135 x y+0.000052673 x^{3}-0.000038232 y^{3}$ (mGal)

Les unités des coordonnées $\mathrm{x}$ et $\mathrm{y}$ utilisées pour ces calculs sont définies d'après le système UTM de la manière décrite auparavant. Les termes en $\mathbf{x}^{2} \mathrm{y}$ et en $\mathrm{x}^{2}$ sont omis parce que leurs coefficients sont plus petits que $10^{6}$.

\section{(4) Moyenne mobile}

Une méthode apparentée aux méthodes anciennes telles que définies par Griffin (1949), mais utilisant en même temps les moyens analytiques numériques et informatiques, est la méthode de la moyenne mobile appliquée et développée au Maroc central hercynien (Frydecki et al., 1985). L'idée principale de cette méthode consiste à construire une grille et à calculer des valeurs moyennes sur les noeuds. Pour chaque point de cette grille sur la superficie étudiée, on calcule une moyenne des valeurs observées autour du noeud, valeurs renfermées à l'intérieur d'une fenêtre (carrée ou circulaire) de dimension choisie. Cette méthode peut avoir l'explication physique suivante: le produit de son application, i.e., une grille ou une carte des valeurs moyennes obtenues, pondérée ou non, dépendant des dimensions des fenêtres, est plus ou moins identique à l'image obtenue de l'aire et de l'altitude, et celle-ci est du même ordre de grandeur que le rayon ou les dimensions de la fenêtre appliquée. Cette méthode présente aussi un important avantage: on peut choisir les dimensions des fenêtres conformément aux dimensions des corps ou objets examinés et à la densité des points de mesure.

Les dimensions du Mont Mégantic sont confinées à un carré de $10 \mathrm{~km} \times 10 \mathrm{~km}$. Afin de pouvoir considérer les paramètres gravimétriques du Mont Mégantic comme anomalies par rapport au champ régional, nous avons choisi quatre dimensions d'essai.

La Figure 8 fait voir les images de la régionale pour des fenêtres mobiles de $10 \times 10,20 \times 20,30 \times 30$ et $40 \mathrm{~km} \times 40 \mathrm{~km}$. Les approximations avec des fenêtres de $10 \mathrm{~km} \times 10 \mathrm{~km}$ (Fig. 8a) et de $40 \mathrm{~km} \times 40 \mathrm{~km}$ (Fig. 8d) sont significativement différentes de celles de $20 \times 20 \mathrm{et} 30 \mathrm{~km} \times 30 \mathrm{~km}$. La première approximation (Fig. 8a) est insuffisamment lissée et trop peu généralisée pour représenter la régionale et la quatrième (Fig. 8d), trop généralisée, ne comporte pas d'anomalie positive dans le secteur sud-est de la région, caractéristique observée sur les autres images (Fig. $8 \mathrm{a}, \mathrm{b}$ et $\mathrm{c}$ ). Les deux approximations de la régionale qui soient acceptables sont donc celles des Figures 8 b et c: fenêtre de $20 \mathrm{~km}$ 


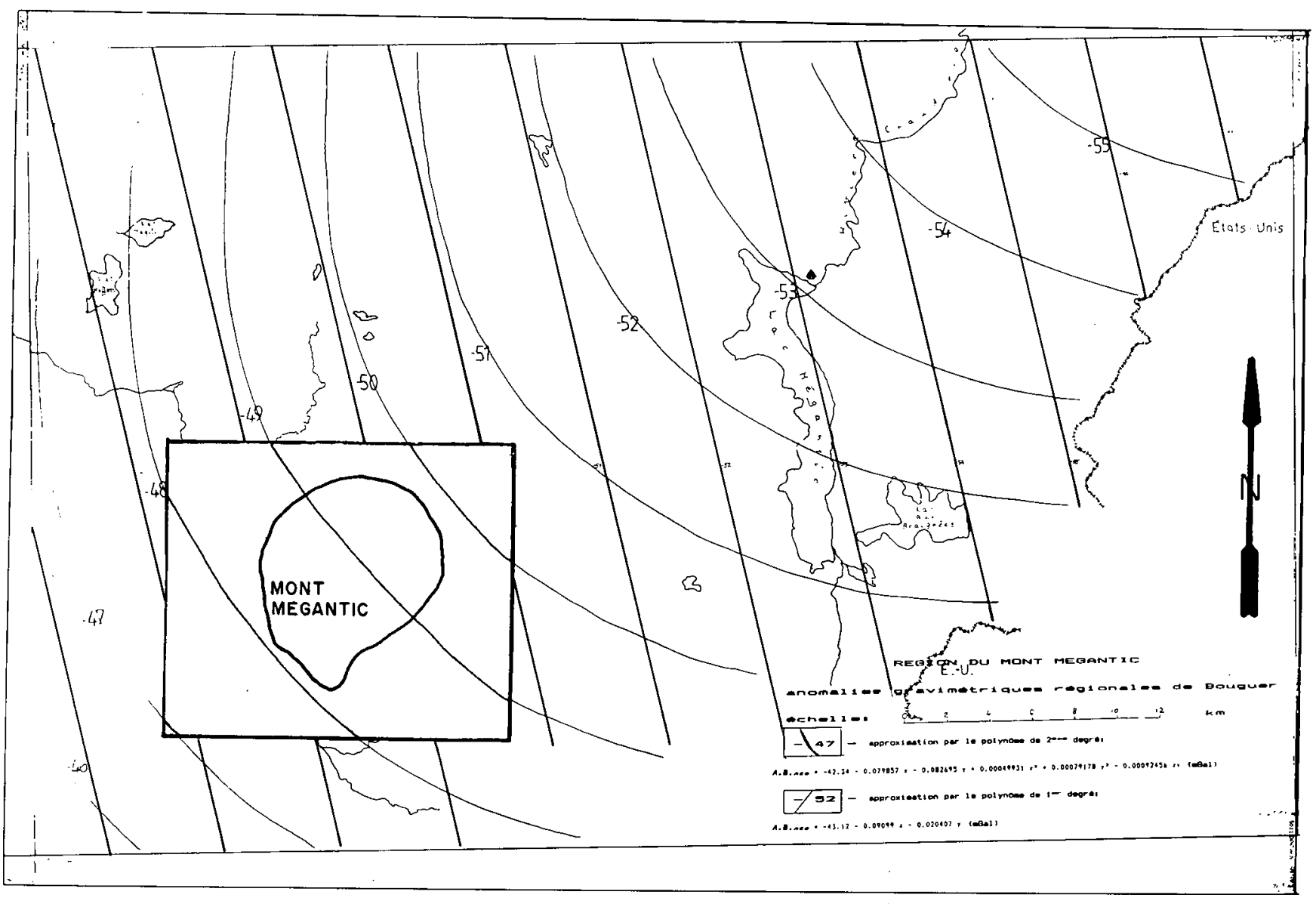

Fig. 5. Approximation des anomalies régionales de Bouguer par le polynôme du $2^{\text {ème }}$ degré superposée à l'approximation du $1^{\text {er }}$ degré dans la région du Mont Mégantic.

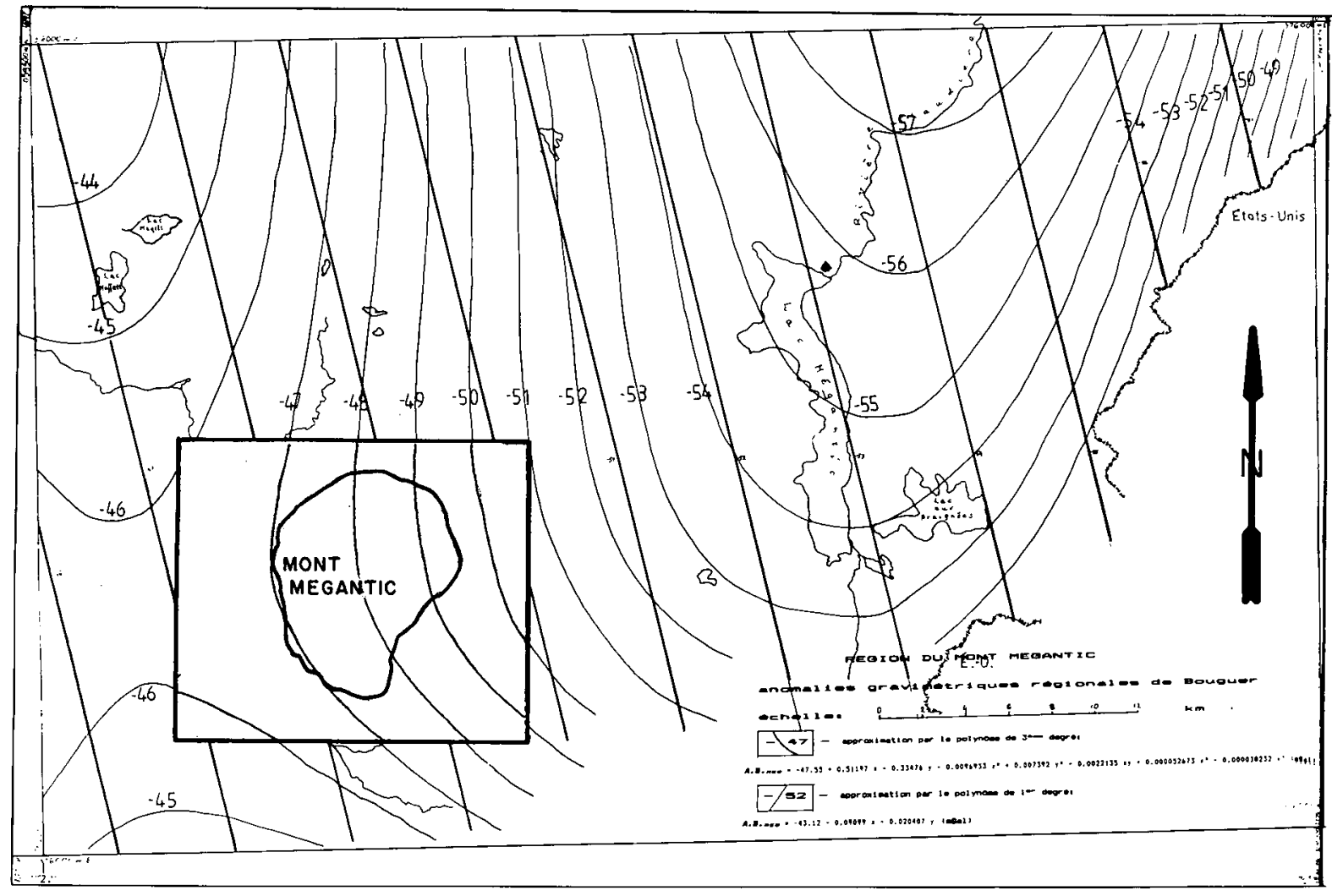

Fig. 6. Approximation des anomalies régionales de Bouguer par le polynôme du $3^{\text {ème }}$ degré superposée à l'approximation du $1^{\text {er }}$ degré dans la région du Mont Mégantic. 

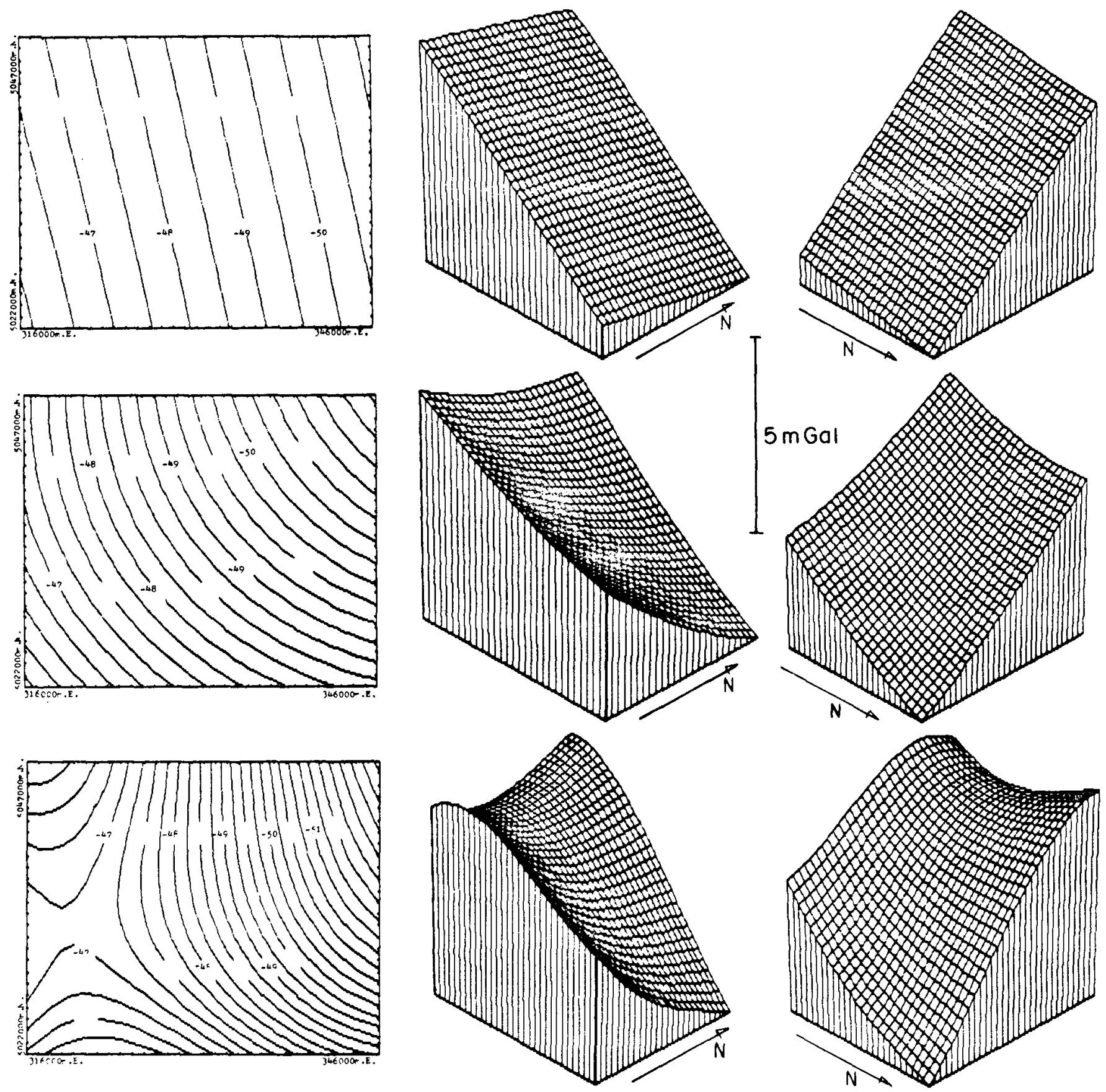

Fig. 7. Comparaison des modèles bi- et tridimensionnels des approximations de $1^{\text {er }}, 2^{\text {ème }}$ et $3^{\text {ème }}$ degré.

$x 20 \mathrm{~km}$ et une seconde $30 \mathrm{~km}$ x $30 \mathrm{~km}$. Le même ensemble de données employé lors des tests avec les polynômes (soit les 111 valeurs supplémentaires de l'anomalie de Bouguer A.B. provenant de la Commission Géologique du Canada) a été utilisée pour comparer ces deux méthodes. Les lignes de contour ont été tracées manuellement et le pas de déplacement de la fenêtre utilisé est de $1 \mathrm{~km}$. L'emploi d'un petit pas de déplacement ne requiert pas nécessairement de pondération du filtre sélectionné. Les résultats de ces applications du champ régional sont présentés à la Figure 9 (pour la fenêtre de $20 \mathrm{~km} \times 20 \mathrm{~km}$ ) et à la Figure 10 (pour la fenêtre de $30 \mathrm{~km} \times 30 \mathrm{~km}$ ). Chaque cas présenté est superposé à l'approximation du $1^{\text {er }}$ degré qui indique une tendance directionnelle incontestable à une échelle globale. Les différences entre ces deux images (Figs. 9, 10) sont facilement discernables. La variante de $30 \mathrm{~km} \times 30 \mathrm{~km}$ est visiblement plus généralisée, surtout pour les parties périphériques. Dans le secteur considéré (celui du Mont Mégantic), la différence des deux représentations s'étale entre 1 et $2 \mathrm{mGal}$. Les directions dans cette partie sont presque les mêmes. Les deux images ne sont pas chargées par des détails; ces deux approximations reproduisent très bien toutes les tendances directionnelles des variations du champ régional.

\section{COMPARAISON DES RESULTATS ET CHOIX DE LA REGIONALE}

La comparaison entre les résultats des approximations par les polynômes de $1^{\text {er }}, 2^{\text {ème }}$ et $3^{\text {ème }}$ degré a été présentée 

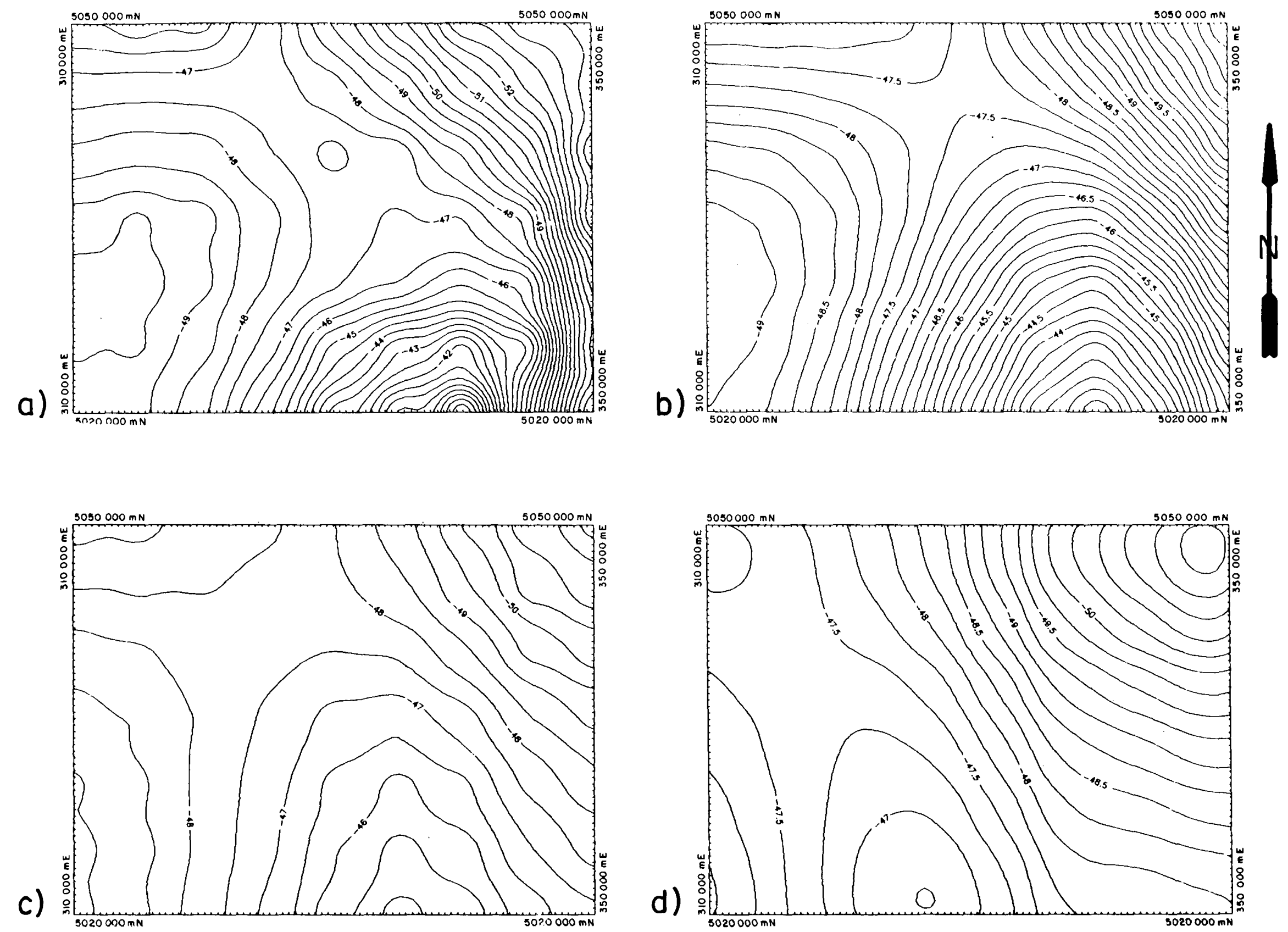

Fig. 8. Anomalie régionale de Bouguer du secteur ouest de la région du Mont Mégantic considérée dans les Figures 8, 9 et 10. Approximation par la méthode de la moyenne mobile avec un pas de décalage de $2,5 \mathrm{~km}$. Le calcul de la moyenne, le lissage et le traçage sont entièrement automatisés. (a) fenêtre de $10 \mathrm{~km} x 10 \mathrm{~km}$; (b) fenêtre de $20 \mathrm{~km}$ $\times 20 \mathrm{~km}$; (c) fenêtre de $30 \mathrm{~km} \times 30 \mathrm{~km}$; (d) fenêtre de $40 \mathrm{~km} \times 40 \mathrm{~km}$. 


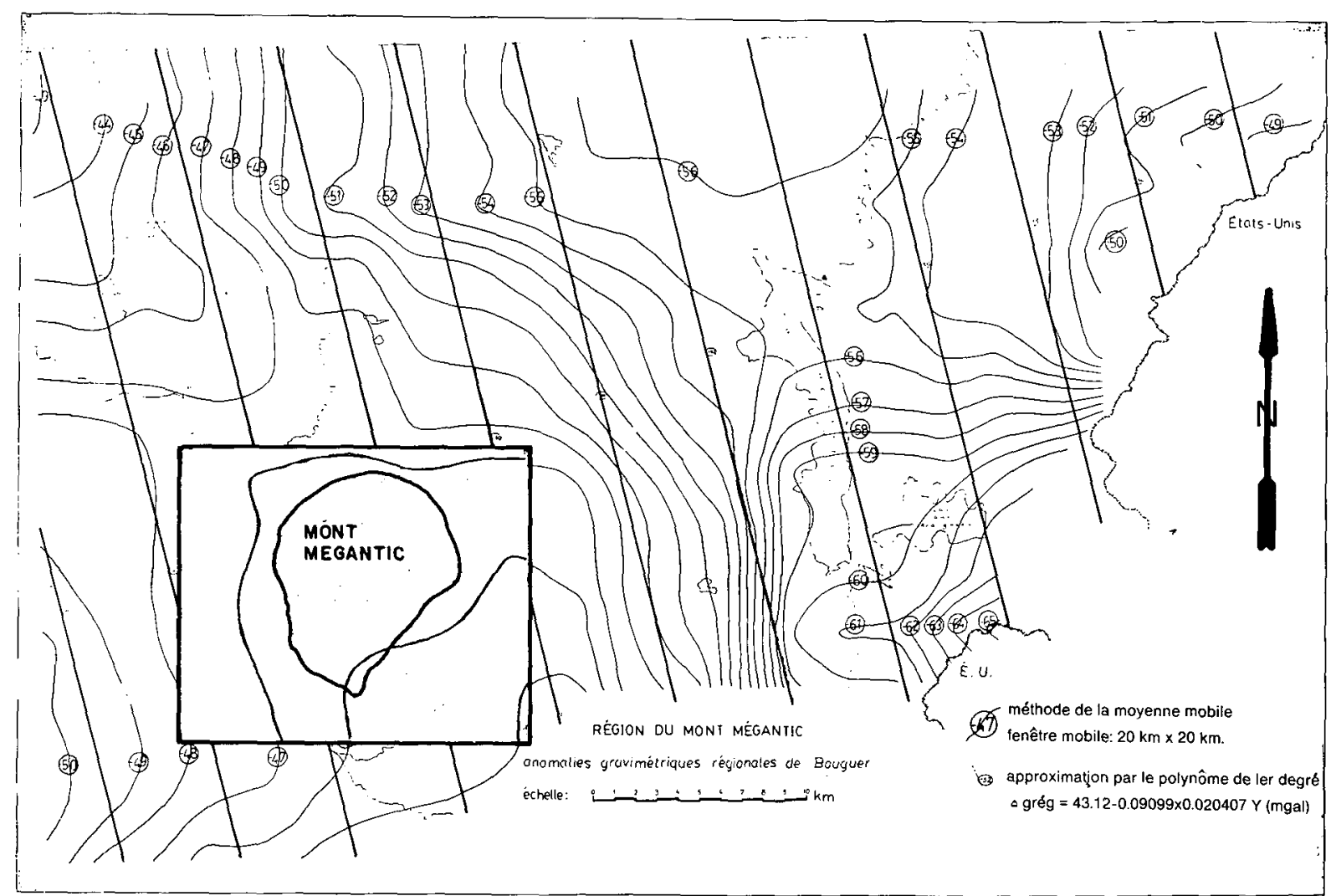

Fig. 9. Approximation de l'anomalie régionale de Bouguer par la méthode de la moyenne mobile (fenêtre de $20 \mathrm{~km} \times 20 \mathrm{~km}$ ) superposée à l'approximation du $1^{\text {er }}$ degré.

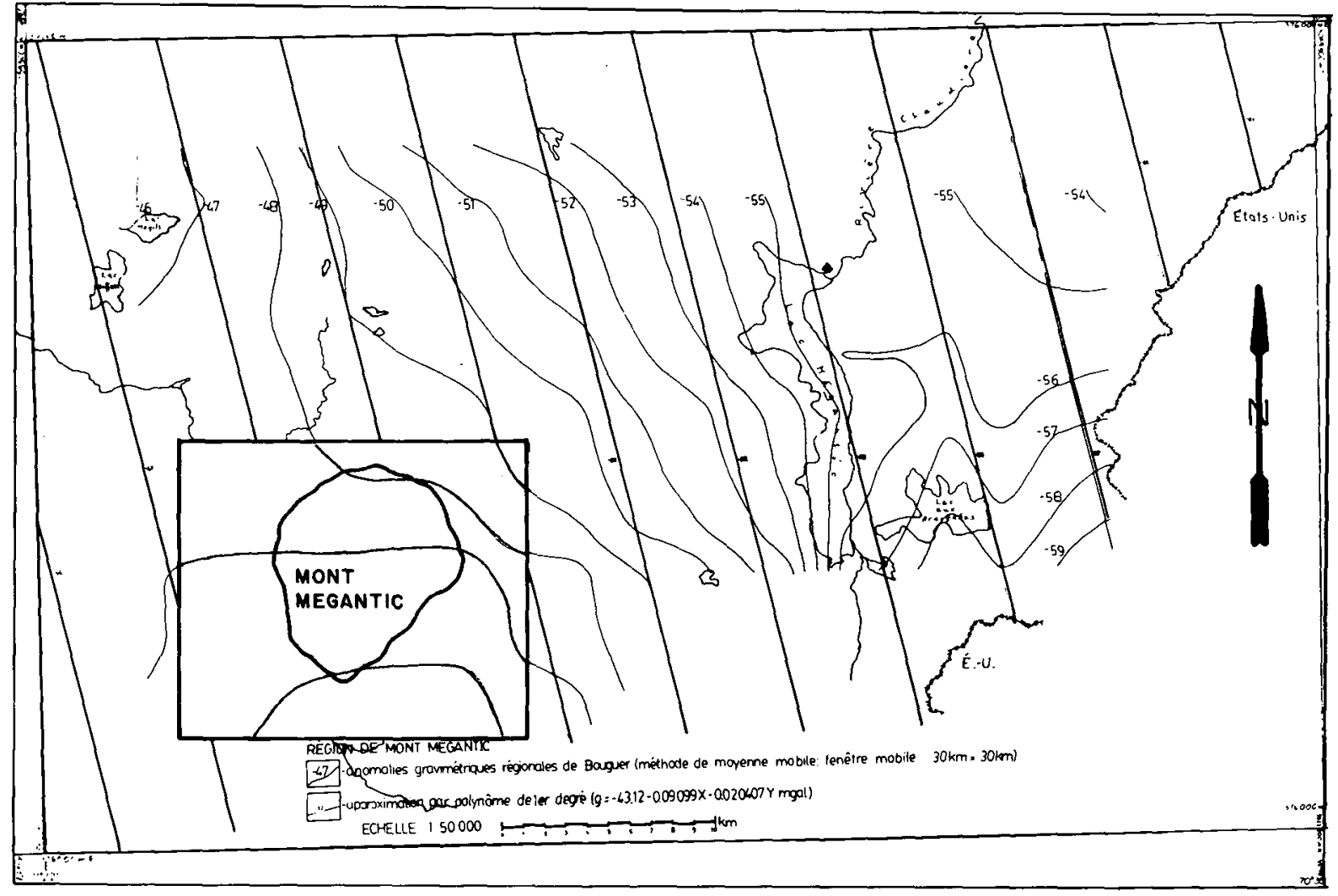

Fig. 10. Approximation de l'anomalie régionale de Bouguer par la méthode de la moyenne mobile (fenêtre de $30 \mathrm{~km} \times 30 \mathrm{~km}$ ) superposée à l'approximation du $1^{\text {er }}$ degré dans la région du Mont Mégantic. 
précédemment. Il reste maintenant à analyser quelle variante de la régionale choisir, i.e., celle de l'approximation de $3^{\text {ème }}$ degré ou bien une de celles obtenues par la méthode de la moyenne mobile. Cette fois, le choix doit être justifié par la dimension de l'objet géologique, l'échelle du levé, i.e., par un critère objectif soit physique soit géologique. Toutes les méthodes de traitement numérique qui donnent comme produit final une formule mathématique ou des coefficients pour une autre formule ont un avantage difficilement négligeable, à savoir facilité du stockage des résultats et de nouveaux traitements. Dans chaque cas, il ne s'agit que d'une formule à conserver et à utiliser. Le problème de la banque des données disparaît. D'après la formule obtenue, chaque configuration possible de la grille, chaque nouveau modèle peut être obtenu simplement en utilisant cette formule sans nécessiter de recours aux données. Même si la mémoire des ordinateurs est volumineuse et peu dispendieuse de nos jours, une formule est plus commode qu'une banque de données. La facilité décrite plus haut représente toutefois un critère mathématique ou plutôt technique.

Malheureusement, la méthode de la moyenne mobile ne possède pas cet avantage; les résultats obtenus par cette voie sont les valeurs dans les noeuds de la grille choisie. Chaque autre configuration de la grille exige un nouveau traitement. Il faut stocker les résultats de ce traitement dans la banque des données, sinon il faudrait effectuer le traitement à nouveau si l'on désire réutiliser les résultats. Les désavantages techniques de cette méthode en comparaison avec la méthodes des polynômes existent, mais la qualité de l'information géologique obtenue joue un rôle décisif en faveur de l'emploi de cette approche. N'étant pas trop pressé par le temps et ne rencontrant pas de problèmes techniques spécifiques, il est préférable d'opter pour la méthode comportant une qualité supérieure d'information et ayant une justification géologique. On a donc choisi pour les travaux de détail qui vont suivre cette étape d'élaboration des données, soit la régionale de Bouguer obtenue d'après la méthode de la moyenne mobile.

Finalement, il faut choisir parmi les deux variantes de fenêtres mobiles appliquées dans les approximations (Fig. 11). La fenêtre de dimension $20 \mathrm{~km} \times 20 \mathrm{~km}$ donne une image presque calme, constante à l'intérieur de l'aire du Mont Mégantic, mais elle devient plus bruyante aux bordures de la carte. La variante de la fenêtre de dimension $30 \mathrm{~km} \times 30 \mathrm{~km}$ est généralement plus lisse, visiblement plus généralisée sur toute la région considérée, surtout pour les parties périphériques. Toutefois, à l'intérieur de l'aire du Mont Mégantic, on observe une petite variation de l'ordre de $2 \mathrm{mGal}$ plus élevée que celle de la fenêtre $20 \mathrm{~km} \times 20$ $\mathrm{km}$. Les directions des variations dans cette partie sont presque les mêmes. En dernière instance, ce sont ces influences de l'extérieur vers l'intérieur ainsi que les critères des surfaces qui ont décidé du choix. La surface de la fenêtre de dimension: $20 \mathrm{~km}$ x $20 \mathrm{~km}$ déborde la surface de l'aire du Mont Mégantic par un facteur de 2 à 3 fois tandis que la surface de la fenêtre de dimension $30 \mathrm{~km} \times 30 \mathrm{~km}$ est environ 10 fois plus grande que celle de l'objet géologique investigué.

L'augmentation des dimensions de la fenêtre diminue effectivement la surface étudiée: l'aire du Mont Mégantic se déplace en effet vers le coin SW de la carte et elle se situe finalcment en bordure de la carte. L'approximation de la régionale avec fenêtre de $20 \mathrm{~km} \times 20 \mathrm{~km}$ est jugée plus représentative en vertu de son aspect plus généralisé. Plus spécifiquement, l'amplitude de l'anomalie positive au sud est de l'aire est moins atténuée et les rayons de courbure des isolignes moins prononcés. Un choix judicieux de la régionale telle que définie par la méthode de la moyenne à l'intérieur d'une fenêtre mobile de $20 \mathrm{~km} \times 20 \mathrm{~km}$ s'impose donc.
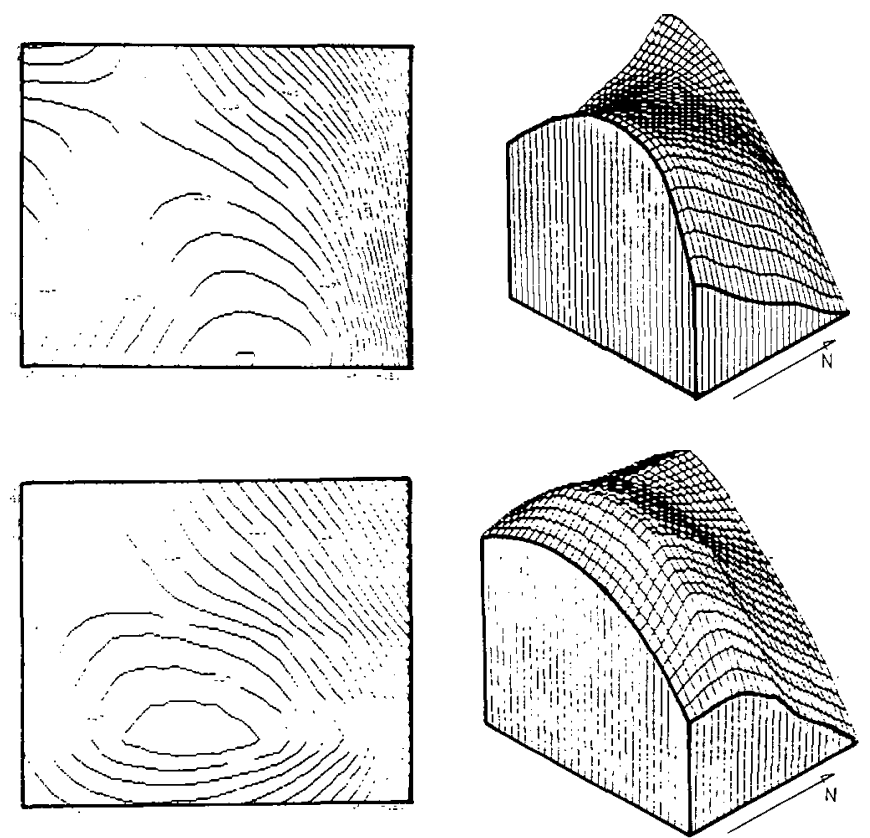
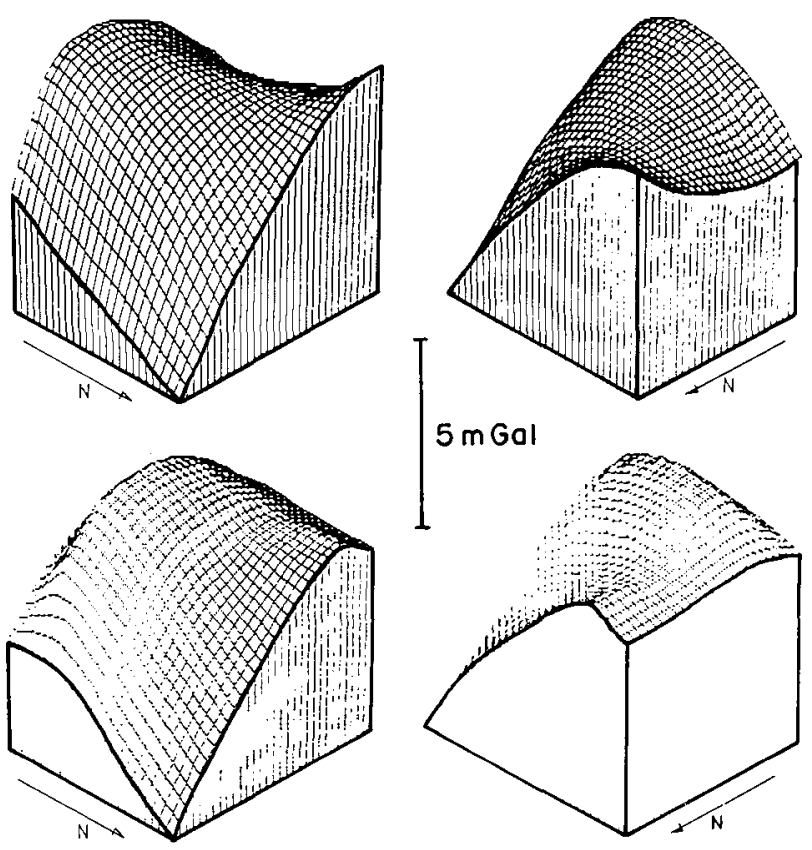

Fig. 11. Comparaison des modèles bi- et tridimensionnels de l'approximation de l'anomalie régionale de Bouguer par la méthode de la moyenne mobile appliquée à la région du Mont Mégantic. En haut: fenêtre de $20 \mathrm{~km} \times 20 \mathrm{~km}$ et en bas: fenêtre de $30 \mathrm{~km} \times 30 \mathrm{~km}$. 


\section{CONCLUSIONS}

Avec les données gravimétriques actuellement disponibles sur et au pourtour du Mont Mégantic, nous avons démontré qu'il est possible d'obtenir une carte (ou des cartes) de l'anomalie régionale adéquate. Un problème courant en géophysique est le suivant: Comment peut-on affirmer qu'une méthode d'interprétation de données géophysiques est meilleure qu'une autre si la structure géologique n'est pas connue ou s'il ne s'agit pas d'un modèle artificiel? La réponse dépend de l'objectif posé au début de l'étude. Les auteurs ont axé le traitement des données sur l'aspect "dimension" des objets géologiques. Conséquemment, les méthodes d'interprétation faisant appel au facteur dimension sont meilleures par définition. Après application et comparaison des deux méthodes, on a choisi la méthode la plus flexible et pour laquelle le choix des paramètres sélectionnés est justifié directement par les dimensions de l'objet investigué. Le choix définitif de la régionale est alors l'approximation par la méthode de la moyenne mobile avec une fenêtre de dimension $20 \mathrm{~km} \times 20 \mathrm{~km}$. Cet article ne prétend pas donner une solution générale au problème relié à la séparation: anomalies gravimétriques régionale et résiduelle mais on a clairement identifié les difficultés inhérentes à ce problème.

\section{REMERCIEMENTS}

Ce travail a été subventionnée grâce à une entente de recherche de la Commission Géologique du Canada (MERC) accordée à M.K. Seguin.

BURTON, R.F. 1931. Commercial granites of Quebec. Part I. South of the St. Lawrence River. Ministère des mines du Québec, Québec (Qué.), Annual Report, Part E.

CLEMENT, P. et DE KIMPE, C.R. 1977. Geomorphological conditions of gabbro weathering of Mount Megantic, Quebec. Canadian Journal of Earth Sciences, 14, pp. 2262-2273.

CHEVE, S.R. 1975. Etude stratigraphique, tectonique, volcanique et métallogénique de la région du lac Mégantic. Ministère des richesses naturelles du Québec, Québec (Qué.), DP-305, 31 p. - 1977. Région de Notre-Dame-des-Bois - Chartierville. Ministère des richesses naturelles du Québec, Québec (Qué.), DPV$512,13 \mathrm{p}$.

. 1978. Région du sud-est des Cantons de l'Est. Ministère des richesses naturelles du Québec, (Qué.), DP-613, 80 p.

DANIS, D. 1984. Géologie du complexe granitique du Mont Mégantic. Ministère de l'Energie et des Ressources du Québec, Québec (Qué.), MB 85-07, 20 p.

FRYDECKI, J., HAMOUDA, M.F., NOWICKA, E., et RHATOUS, A. 1985. Traitement de données géophysiques à partir d'une méthodologie non classique: application à la région de Moumou - Moulay Bou Azza (Maroc central hercynien). Actes des Joumées Géologiques et Minières Marocaines, Rabat, 22 p.

FRYDECKI, J., SEGUIN, M.K., et ROY, S. 1987. Modélisation gravimétrique de l'intrusif du Mont Mégantic. Actes du colloque de géophysique. $55^{\text {tme }}$ congrès de l'ACFAS, Ottawa.

GRIFFIN, W.R. 1949. Residual gravity in theory and practice. Geophysics, 14, pp. 39-56.

MCGERRIGLE, H.W. 1934. Mount Megantic area, southeastem Quebec and its placer gold deposits. Quebec Department of Mines, Annual Report, Part D.

NETTLETON, L.L. 1954. Residuals, regionals and structures. Geophysics, 19, pp. 1-22.

REID, A.M. 1960. Rapport préliminaire sur la géologie du Mont Mégantic, comtés de Compton et de Frontenac. Département des mines du Québec, Québec (Qué.), R.P. 433, 6 p.

- 1976. Géologie du Mont Mégantic. Ministère des richesses naturelles du Québec, Québec (Qué.), ES-25, 59 p.

ROY, S. et SEGUIN, M.K. 1986. Etude gravimétrique du Mont Mégantic. Actes du colloque de géophysique. $54^{\text {ème }}$ congrès de l'ACFAS, Montréal, pp. 30-40.

SAXOV, S. et NYGAARD, K. 1953. Residual anomalies and depth estimation. Geophysics, 17, pp. 913-924.

SEGUIN, M.K. et FRYDECKI, J. 1989. Densités et anomalies gravimétriques de l'intrusif du Mont Mégantic, Geoexploration, 26, pp. 33-46.

SEGUIN, M.K. et ST-HILAIRE, B. 1985. Magnétochronologie des formations rocheuses du Mont Mégantic et des environs, Québec méridional. Canadian Journal of Earth Sciences, 22, pp. 487-497. 